\title{
Pratiques spatiales transnationales et stratégies de mobilité des commerçantes tunisiennes
}

\section{Camille Schmoll}

\section{(2) OpenEdition \\ 1 Journals}

Édition électronique

URL : https://journals.openedition.org/remi/2352

DOI : $10.4000 /$ remi.2352

ISSN : $1777-5418$

Éditeur

Université de Poitiers

\section{Édition imprimée}

Date de publication : 29 mai 2005

Pagination : 131-154

ISBN : 2-911627-39-3

ISSN : 0765-0752

\section{Référence électronique}

Camille Schmoll, «Pratiques spatiales transnationales et stratégies de mobilité des commerçantes tunisiennes », Revue européenne des migrations internationales [En ligne], vol. 21 - n 1 | 2005, mis en ligne le 04 septembre 2008, consulté le 15 avril 2022. URL : http://journals.openedition.org/remi/2352 ; DOI : https://doi.org/10.4000/remi.2352

Ce document a été généré automatiquement le 14 avril 2022.

(c) Université de Poitiers 


\title{
Pratiques spatiales transnationales et stratégies de mobilité des commerçantes tunisiennes
}

\author{
Camille Schmoll
}

1 Dans l'espace euro-méditerranéen, les évolutions récentes du phénomène migratoire sont marquées par l'intensification et la diversification des pratiques de circulation, parmi lesquelles un commerce transfrontalier à l'initiative de migrants maghrébins (Simon, 1990 ; Tarrius, 1992 ; Missaoui, 1995; Péraldi, 2001; Battegay, 2003). Cette activité économique, qui met en relation des lieux distants et complémentaires, a donné naissance à un vaste espace transnational, constitué de places marchandes, de lieux d'achat et de revente, mais aussi de routes supports aux déplacements des migrants commerçants (Aslafy-Gauthier, 2002). Au cours des années 1980, la ville de Naples est devenue un pôle d'approvisionnement important dans cette géographie des mobilités commerçantes.

2 Parallèlement, les flux de travailleurs maghrébins orientés vers l'Europe ont connu une féminisation, qui se traduit notamment par le développement des migrations de femmes seules, rompant avec l'image traditionnelle de l'épouse et de la mère de famille, suivant son mari dans le cadre du regroupement familial (Campani, 1995 ; Ramirez, 1999 ; Zontini, 2002). Cette féminisation des flux de travailleurs reflète un mouvement plus général de participation croissante des femmes à la sphère publique et professionnelle dans les pays du Maghreb (Moghadam, 1998; Jomni, 2000 ; Bekkar, 2004).

Or, si ces deux aspects des dynamiques migratoires, le développement des circulations commerciales et celui des migrations de travail féminines, ont fait séparément l'objet de travaux, la féminisation des circulations commerciales dans l'aire méditerranéenne est encore un phénomène assez peu connu. Cet article, dont l'objectif est d'attirer l'attention sur cet aspect de la migration féminine, s'appuie sur une recherche réalisée auprès d'un groupe de femmes tunisiennes, originaires de la ville de Sousse, qui pratique de fréquents voyages d'achat à Naples. Bien qu'il s'agisse d'un micro-réseau, le 
cas de ces femmes est significatif, car il renvoie à une réalité circulatoire en pleine expansion : des collectifs féminins similaires partent de nombreuses villes et se croisent sur chaque bateau transitant de Tunisie en Italie. Surtout, l'Europe ne capte qu'une minorité de ces flux. La plupart des circulantes tunisiennes s'approvisionnent en Libye et au Maroc, en Turquie et en Syrie. Il faut donc considérer cet exemple comme révélateur d'un mouvement de circulation plus ample, qui couvre l'ensemble du bassin méditerranéen.

Un autre indice de cette généralisation des circulations féminines est la diversité des situations sociales de celles qui transitent sur les routes du commerce. La circulation n'est plus le privilège des migrantes installées durablement en Europe, ni même des seules commerçantes. Naples est à la fois un lieu d'achat pour des entrepreneuses avisées et une destination de "shopping transnational ${ }^{1}$ pour des voyageuses occasionnelles. La distinction entre ces deux figures peut même parfois sembler artificielle, car les commerçantes tirent également profit de leurs voyages en Europe pour effectuer des achats destinés à leurs besoins personnels et à leur entourage. C'est pourquoi, si ce texte est consacré aux femmes qui pratiquent le commerce de façon régulière, il soulève également des questionnements quant à l'impact des circulations sur les pratiques de consommation au sein des sociétés maghrébines, et particulièrement dans les classes moyennes. Surtout, cette imbrication entre commerce et consommation individuelle montre bien que le voyage n'est pas une simple activité professionnelle, visant uniquement à générer du profit, mais bien une expérience complexe et riche en signification, à l'instar de formes de migration plus durables.

5 Les séjours d'achat des femmes qui ont été interrogées ont lieu en moyenne une fois par mois, et durent de quatre à dix jours ${ }^{2}$. Femmes dans des places d'hommes et souvent femmes d'âge mûr ${ }^{3}$, elles ne correspondent guère à la représentation habituelle du migrant maghrébin. La spécificité de leur pratique migratoire, faite de vaet-vient continus entre ici et là-bas, interpelle : que se joue-t-il en termes économiques, mais aussi relationnels, dans ces fréquents voyages? La circulation commerciale renforce-t-elle ou affaiblit-elle leur « subordination de genre » (Bjeren, 1997) ? Quels sont les obstacles ou, inversement, les «savoir-circuler » spécifiques à la circulation féminine (Tarrius, 1992) ? Dans quelle mesure leur identité de genre, sur des routes et des places essentiellement contrôlées et fréquentées par des hommes, contribue-t-elle à orienter leurs pratiques socio-spatiales?

6 Pour répondre à ces questions, il convient à la fois de considérer ces femmes sous l'angle de leurs multiples positions sociales (Moore, 1994), mais aussi de tenir compte des différents lieux qu'elles parcourent et qui composent leurs espaces de vie. C'est pourquoi de multiples échelles (l'espace domestique, le quartier, la ville, l'espace de la traversée) sont articulées dans ce texte (Silvey, 2004). On verra que pour ces femmes, la circulation est mise au service de formes de promotion sociale et d'autonomisation. Toutefois, parce qu'elle engage la mixité dans un monde où les espaces féminins et masculins sont souvent séparés, la mobilité spatiale est l'objet de multiples négociations et génère des formes d'organisation spécifiquement féminines. 


\section{Profils de la migration féminine et circulations commerciales}

\section{De nouvelles figures de la migration féminine}

7 La participation des femmes à la sphère productive dans le contexte des migrations internationales, après avoir été longtemps négligée, est de plus en plus connue et documentée. Deux figures émergent de la littérature contemporaine sur la migration et le commerce féminins.

8 Des travaux récents, notamment ceux concernant les « nouvelles migrations » en Europe du Sud, mettent l'accent sur l'importance des femmes migrant seules, qui s'insèrent dans des niches particulières, dans le cadre d'une division sexuée et ethnique du marché mondial du travail (Anthias, Lazaridis, 2000). Ces niches sont principalement le travail domestique, la prostitution et les métiers du «divertissement" (" entertainment»). Les femmes répondent alors à une demande spécifique de la part des sociétés d'accueil, au sein d'un marché du travail segmenté. Peu de ces études sont consacrées aux femmes maghrébines. Toutefois, les rares travaux concernant les femmes primo-migrantes originaires du Maghreb mettent l'accent sur la nécessité de prendre en considération la relation qu'entretient la femme à son pays d'origine pour interpréter son itinéraire. Ils montrent ainsi que les femmes maghrébines qui émigrent seules ne laissent pas derrière elles de situations maritales: elles sont en général célibataires, veuves, divorcées ou répudiées. L'émigration est alors vécue comme un moyen de partir sur de nouvelles bases, de se construire un nouveau projet de vie au pays d'accueil (Ramirez, 1999, 2002 ; Zontini, 2002).

9 En parallèle, une autre figure émerge de la littérature sur la migration et concerne le commerce féminin: la commerçante internationale africaine, souvent associée à la Nana-Benz qui est la grande commerçante de Lomé, cette « Togolaise, revendeuse de pagnes roulant en Mercedes, devenue sur le continent africain le symbole de la réussite féminine» (Bredeloup, 2001 : 83 ; voir aussi Humarau, 1997). L'importance de cette figure, qui a été transposée au champ des migrations vers l'Europe, renvoie à la tradition de commerce féminin en Afrique subsaharienne. Souvent, des ruptures conjugales dans la vie de ces femmes (veuvage, divorce) sont l'occasion de s'affranchir de contraintes domestiques tout en gagnant en autonomie sur le plan économique (Sengel, 2000 ; Bredeloup, 2001). La commerçante africaine, parfois collectionneuse d'hommes, joue alors de sa sensualité et de ses différents mariages pour développer des stratégies d'ascension sociale.

\section{La circulation commerciale féminine, entre continuité et reformulation des rôles féminins}

10 Les femmes commerçantes rencontrées s'éloignent de ces catégories de migrantes. Elles s'apparentent à une autre figure de la migration féminine, celle de la commerçante maghrébine, tirant profit d'espaces distants pour mettre en œuvre des stratégies de promotion sociale. Cette figure a été signalée par Yeza Boulahbel (1996) dans le cas des Algériennes, et plus récemment par Saadia El-Hariri (2003), pour les Marocaines. Elles montrent comment les épouses de migrants maghrébins, loin d'être, comme l'exige la représentation commune, des actrices passives, doivent être vues comme des agents 
"autonomes et spécifiques» capables de mettre en œuvre des stratégies d'accumulation personnelle. Toutefois, il s'agit encore d'épouses de Maghrébins installés en France, qui pratiquent le commerce en tant qu'activité complémentaire. En revanche, Michel Péraldi a identifié des cohortes de femmes, domiciliées en Algérie, qui effectuent régulièrement des voyages d'achat en Turquie (Péraldi, 2001). Les femmes tunisiennes évoquées ici s'apparentent à cette dernière figure. Il ne s'agit plus de migrantes installées en Europe, mais bien de femmes issues de différentes couches sociales des sociétés maghrébines, qui se rendent à l'étranger le temps de voyages de commerce.

11 À la différence des travailleuses du sexe et des domestiques installées en Europe, ces femmes ne répondent pas à une demande de travail émanant des sociétés européennes, mais s'inscrivent davantage dans les évolutions du marché du travail au Maghreb, qui se caractérisent par une féminisation de certaines activités d'ordinaire réservées aux hommes comme le commerce (Jomni, 2000). À cet égard, elles se situent au cœur des mutations actuelles des sociétés maghrébines.

Le phénomène n'est en réalité pas si récent. On signale, dès les années 1960, des va-etvient de commerçantes entre l'Italie et la Tunisie. La nouveauté semble plutôt résider dans leur généralisation et dans la conquête progressive de la part des femmes de nouveaux espaces commerciaux. Du point de vue des espaces d'achat, elles fréquentent désormais de nombreuses places marchandes en Europe et en Méditerranée, certaines poussant leurs déambulations commerciales jusqu'en Asie. Les espaces de vente ont également connu un élargissement significatif, marqué par un glissement du privé vers le public. En effet, à la différence de l'Afrique sub-saharienne, on ne trouve guère, dans les pays du Maghreb, de tradition de commerce féminin dans les espaces publics. Autrefois confinée dans l'intimité de l'espace domestique, la revente s'effectue désormais de plus en plus dans les souks et dans les boutiques. Si elle est particulièrement affirmée dans le cas des circulantes commerciales, cette dynamique de conquête de l'espace public témoigne d'une entrée généralisée des femmes dans la sphère professionnelle, dans les économies formelle et informelle, qui s'assortit d'une diffusion de la mixité des genres dans l'espace urbain ${ }^{4}$ (Berry-Chikhaoui, 2000 ; Jomni, 2000). Cette négociation pour s'extraire de l'espace domestique (sans toutefois abandonner totalement la vente à domicile) s'effectue progressivement, comme l'illustre le témoignage de Hayet, 46 ans, qui raconte comment elle a conquis l'espace du souk (le marché hebdomadaire) d'abord en famille, puis seule :

"Avant, la femme ne pouvait pas sortir au souk, c'était défendu, elle n'était pas acceptée, donc elle vendait chez elle. Ça fait à peu près deux ans que "ça passe". Avant, surtout au début de mon commerce, je vendais à la maison. Je travaille au souk depuis huit ans avec mon mari et mon fils. Depuis deux ans, j'y vais seule ».

Les produits commercialisés par ces femmes concernent principalement le secteur du mariage: habillement, mais aussi services de table, bijoux, tapis, parures matrimoniales. Ce dernier produit tient une place tout à fait à part dans les pratiques de consommation des sociétés maghrébines et dans les circulations commerciales entre le Maghreb et l'Italie: énormes valises de carton, armées de plusieurs tiroirs, les parures matrimoniales contiennent du linge de maison, mais aussi quelques pièces de lingerie ou encore des accessoires de beauté. Souvent commercialisées exclusivement au Maghreb ou dans les boutiques de bazar maghrébines d'Europe, ces valises constituent la parfaite panoplie du jeune couple. Celles de meilleure qualité, brodées à la main, de dentelle ou de soie, sont toujours de production italienne, et constituent un cadeau de mariage idéal. À cet égard, les commerçantes s'inscrivent dans la tradition 
selon laquelle tout ce qui touche à la préparation et à l'organisation des mariages est de leur compétence (Troin, 1975 ; Boulahbel, 1996).

Une autre raison pour laquelle ces femmes se détachent des figures de la migration évoquées plus haut est leur ancrage et leur présence physique fréquente au foyer. La migration n'entraîne guère de rupture dans la vie familiale et conjugale, dans la mesure où une grande partie des investissements et des décisions liées à la migration font l'objet d'une négociation au sein du foyer.

\section{Un dessein de mobilité sociale et d'autonomisation, négocié dans le cadre du foyer}

\section{Différents registres des motivations}

15 Quelle signification les femmes accordent-elles à la circulation? Comment justifientelles leur choix de migrer? Comment les bénéfices de leur activité sont-ils réinvestis? Les commerçantes qui se rendent à Naples sont issues de générations différentes, mais ont toutes pour point commun d'être des femmes mariées, à l'exception des filles qui suivent leur mère. Leurs maris sont petits fonctionnaires ou commerçants, ouvriers, employés, ou chômeurs. Elles insistent toujours, en premier lieu, sur la nécessité de subsistance quotidienne à laquelle répond l'exercice du commerce. De fait, elles constituent toujours le premier revenu de leur ménage, même si leur rôle est parfois minoré dans les entretiens. Les déclarations de Zahra, âgée de 55 ans et mère de 4 enfants, montrent que la migration correspond à une nécessité matérielle forte, et s'inscrit d'emblée dans le cadre d'un projet conjugal :

« Tout le monde va te raconter la même histoire, car ce sont les mêmes conditions qui font faire du commerce, personne ne peut nous en empêcher parce qu'il y a une nécessité, une nécessité commerciale, qui nous oblige à sortir, à s'entraider, l'homme avec la femme, pour confronter la faim ».

Cependant, le choix de migrer ne correspond pas uniquement à une nécessité pure et dure, comme en témoigne la diversité des milieux sociaux de ces femmes, dont certaines proviennent de classes sociales favorisées. La pratique du commerce participe plutôt d'un projet familial d'ascension sociale, les revenus dégagés permettant d'accroître le bien-être général de la famille.

Les femmes investissent parfois leurs revenus dans des projets immobiliers et commerciaux: la construction d'une maison, l'achat d'une boutique, ou encore la réalisation de petites opérations de spéculation immobilière sont fréquents, même si les formes d'accumulation demeurent généralement limitées. Elles ne dévalorisent guère leur activité, mais n'ont pas l'intention de la transmettre, du moins de façon définitive, à leurs enfants auxquels elles s'attachent à assurer un avenir meilleur : les études et la dot, pour les filles, constituent alors les deux grands domaines d'investissement. Dans les maisons des commerçantes, une pièce est dévolue à la préparation du trousseau des filles, même si elles sont encore très jeunes car, selon les dires de certaines, «le trousseau d'une fille c'est son honneur » : la question du trousseau, emblématique, met en évidence les contradictions et tensions dans les pratiques de ces femmes, entre reproduction de certains clichés de genre et souhait d'émancipation pour leurs filles. 
18 La pratique du commerce est également mise au service de stratégies de reconnaissance sociale. La circulation transnationale permet la construction d'une nouvelle personnalité, à travers les pratiques de consommation. Les femmes commerçantes sont représentatives d'une classe moyenne qui émerge en Tunisie et se caractérise par son engouement pour la mode italienne. Elles utilisent ainsi la consommation de produits italiens à des fins de distinction et insistent sur la " faim » de produits occidentaux qui les pousse à ne jamais arrêter les voyages. La migration permet de combler des aspirations (des nécessités de consommation, un rythme de vie) qui ne sont pas autrement possibles :

«Ma sœur était fiancée. Elle allait se marier, raconte Soumaya (24 ans). Pendant deux mois, on restait trois à quatre jours ici, et on refaisait le voyage! Chacune avait une destination bien déterminée : j'ai été trois fois en Italie parce qu'il y avait de l'électroménager, les accessoires de maison, parce qu'elle voulait des objets bien déterminés, des prises, des miroirs et tout... Puis j'ai été en Turquie pendant une semaine. Elle m'a rejoint pour terminer. Nous sommes allées partout !».

De la même façon, Sabrina (25 ans), quand elle décrit les voyages de commerce de sa mère en Italie, insiste sur le fait qu'il s'agit avant tout d'une sorte de shopping nécessaire au maintien d'un certain standard de vie :

«La première fois qu'elle est allée en Italie elle a fait un petit shopping et on a commencé à avoir un peu de confort, alors elle a refait l'expérience, elle a continué jusqu'à maintenant. Avant on a construit la maison, on a acheté une voiture, on a fait grandir les enfants... mais non, maintenant, quand elle fait du commerce, c'est rien que pour nous ramener tout pour être bien. Parce que nous, maintenant, on ne peut plus utiliser un produit tunisien. C'est plus que l'habitude! Tu fais la comparaison, la comparaison commerciale aussi parce que c'est pas le même prix ici qu'à l'étranger ${ }^{5}$, alors tu dis c'est plus rentable que j'aille faire mon shopping d'hiver ou d'été plutôt que je reste ici ».

20 Le déménagement de l'appartement à la villa est vécu comme un acte de promotion particulièrement important, qui symbolise dans les parcours le changement de statut ${ }^{6}$. Loin d'être délaissé par la migrante, l'espace domestique, qui est à la fois espace d'investissement et de distinction sociale, espace privé et espace de vente, lieu de consommation et lieu de représentation, est profondément transformé. Son aménagement permet d'afficher la réussite de l'entreprise de mobilité sociale. Le salon dit "européen", qui jouxte parfois un salon "traditionnel», se caractérise par l'importance des meubles et objets rapportés d'Italie. Il témoigne du double ancrage de ces femmes, entre ici et là-bas, et des changements que les voyages introduisent dans leur vie.

21 La migration fonctionne aussi comme une façon d'affronter l'instabilité du pays de départ. Pour reprendre Douglas Massey, ce n'est pas la pauvreté au sens propre, mais bien la précarité, l'absence de sécurité sociale (et la migration fonctionnerait alors comme une assurance permettant d'affronter les mauvaises conjonctures) qui permettent d'expliquer les formes et modes de la migration actuelle, notamment leur caractère non définitif mais aussi le fait qu'elles proviennent majoritairement de pays dits en transition, et non des plus désavantagés économiquement (en Europe, le Maghreb et les Pays d'Europe centrale et orientale; aux États-Unis, le Mexique). À l'instar de ce qui s'est produit dans d'autres pays en transition, dans les PECO à la suite de la chute des régimes socialistes par exemple, la migration commerciale des femmes tunisiennes permet de pallier les soubresauts et crises éventuelles de l'économie nationale, dans un contexte d'incertitude. Par exemple, la crise du tourisme ${ }^{7}$ qui a suivi 
l'attentat de Djerba du 11 avril 2002 a été durement ressentie par les classes moyennes tunisiennes. Le commerce, bien que les pouvoirs d'achat aient chuté, a pu permettre à ces femmes d'amoindrir le choc.

Le choix de migrer est également l'instrument d'une stratégie d'autonomisation. Il n'est pas anodin que les sociétés migrantes et commerçantes mobilisent de jeunes hommes, souvent des cadets, et des femmes. Ce sont eux « qui forment, dans les sociétés lignagères et patriarcales maghrébines les surnuméraires et les dominés» (Péraldi, 2005 sous presse). La migration, tout comme la sortie au souk, sont des formes d'affirmation de soi dans la mesure où elles permettent de s'extraire, socialement et spatialement, du foyer. Elles permettent de se tracer une trajectoire « qui n'est pas définie uniquement par la famille et le mari " (Massey, Mc Dowell, $1994: 180$ ), pour se négocier une nouvelle importance dans la société de départ, notamment un pouvoir de décision financier. Bien qu'elles puissent, devant les difficultés liées à la conjoncture actuelle, témoigner d'une certaine lassitude et d'une grande fatigue, les femmes commerçantes expriment clairement un goût du voyage, qui témoigne de la sensation d'autonomie que leur procure leur activité. Elles aiment leur métier, dont elles signalent qu'elles ne pourraient se passer. La métaphore de la maladie ou du virus est régulièrement utilisée pour le qualifier. Par exemple, Hafida, la fille d'Hayet, déclare au sujet de sa mère : "si elle ne part pas, elle étouffe, elle bouffe plus, elle tombe malade... ». Les termes utilisés pour s'auto-désigner, "visiteuses", "voyageuses", "touristes ", jamais immigrées, rarement commerçantes, dépassent largement les limites du vocabulaire du commerce et témoignent de ce goût du voyage. L'auto-dérision est également très présente dans leur propos. Ainsi, quand il sera demandé à ces femmes, au début de l'enquête, combien elles sont à exercer cette activité dans leur ville, elles répondent, dans un éclat de rire et s'inspirant librement des Contes des Mille et une Nuits : «Ali Baba et les quarante voleurs, tu connais? ?.

De ces quelques fils tirés à grands traits de l'écheveau des motivations sociales de ces femmes, on peut comprendre que différents "registres des nécessités", imbriqués, coexistent sans nécessairement entrer en contradiction (Mozère, 2002). L'affirmation de Mark Granovetter selon laquelle "les individus ont non seulement des objectifs économiques, mais recherchent également la sociabilité, la reconnaissance d'autrui, le statut et le pouvoir » dans leurs actions économiques prend ici tout son sens (Granovetter, 2000 : 112).Au-delà d'une simple nécessité économique, d'autres objectifs apparaissent dans les motivations de ces femmes: la circulation permet de confronter des conjonctures difficiles et de mettre en œuvre des stratégies de mobilité sociale à plus ou moins long terme, pour elles-mêmes et pour leurs proches. Elle s'inscrit dans le cadre d'une économie domestique: la famille et le foyer sont d'importants domaines d'investissement. Cependant, la circulation est également, pour les femmes, un outil d'autonomisation, comme en témoigne leur entrée progressive sur le souk et les investissements qu'elles réalisent pour améliorer leur commerce, ainsi que le goût dont elles témoignent pour cette pratique. C'est donc une triple motivation, à la fois économique, domestique et individuelle, qui ressort du discours et des pratiques de ces femmes, même si c'est probablement la «part familiale » de ces motivations qui permet d'expliquer la bienveillance de ceux qui restent envers ces voyageuses.

Dans les causes officielles du voyage, une autre motivation est fréquemment évoquée : leurs maris ne pourraient pas se charger des voyages, car, en tant qu'immigrants potentiels, il leur serait beaucoup plus difficile d'obtenir un visa pour l'Europe. Les femmes, en particulier les femmes mûres, attireraient peu l'attention: elles 
obtiendraient facilement les documents nécessaires à leur entrée sur le territoire italien. De cette façon, ce qui, dans la société de départ, a pu constituer un handicap à la mobilité des femmes (simplement être une femme) devient un atout, puisqu'il confère un pouvoir migratoire dont elles tirent profit pour voyager. Cette motivation prend aujourd'hui, avec le durcissement des politiques d'entrée et des contrôles migratoires, une signification certaine. Cependant, il convient de nuancer cette explication car nombre de ces femmes ont commencé à commercer bien avant que le visa pour l'Italie ne soit imposé. Il faut donc l'interpréter comme une sorte d'alibi, une façon de dédouaner le mari de son devoir de subvenir aux besoins de la famille et de neutraliser la "connotation de trahison et de transgression associée au travail à l'extérieur" (Ramirez, 1999).

\section{Le mari, présent et en retrait}

Dans les sociétés maghrébines, en effet, il pèse un parfum de prohibition sur la circulation féminine. Le commerce à la valise est non seulement considéré comme une activité peu légitime bien que tolérée (Péraldi, 2001), mais surtout, il s'agit d'une activité à connotation masculine. Par ailleurs, dans le cas des commerçantes tunisiennes, la femme est le premier soutien de famille. Or, traditionnellement, la rémunération du commerce féminin demeure le bien personnel de la femme. Il est, dans une certaine mesure, honteux pour l'homme d'avoir à compter avec les revenus de sa femme dans les dépenses quotidiennes (Boulahbel, 1996). C'est pourquoi la décision du départ fait l'objet d'une intense négociation. Comment se prend et se mûrit cette décision? Comment concourt-elle à une redéfinition des rôles dans les sphères privée et professionnelle?

La décision de la migration est prise d'un commun accord entre mari et femme. La paix du ménage est, en effet, la condition du départ. Il s'agit de négocier les transformations sans rupture, sous couvert de continuité, même si dans les faits, la migration introduit des bouleversements dans la vie familiale et notamment dans la capacité des femmes à prendre des décisions.

La participation du mari à l'activité commerciale est essentielle. Pour reprendre le vocabulaire d'Erving Goffman (1998), elle sanctionne en quelque sorte son aval et lui permet de "garder la face », d'être fidèle au code d'honneur. Quant à la femme, elle sait témoigner de sa "déférence » : le mari détient parfois les carnets de crédit, se charge des aspects administratifs ou bien des comptes. Quand il en a la possibilité et que sa femme lui demande, il effectue même quelques voyages. Il l'assiste souvent dans les moments publics de la vente, c'est-à-dire dans la boutique ou au souk. Il peut également jouer un rôle de prête-nom. Hayet, par exemple, constitue en 1999 une société d'import-export et demande à son mari d'être son associé. De fait, il ne prend aucune décision dans la gestion des affaires de sa femme :

«Mon associé c'est mon mari, mais il ne travaille pas avec moi ! Sur les papiers c'est mon associé, mais c'est moi qui fait tout ».

Toutefois, la participation du mari n'est pas qu'une façon de garantir son aval : l'appui sur des ressources familiales est nécessaire à la réussite de l'entreprise. Chaque membre de la famille, mari, enfants (quand ils sont adolescents ou jeunes adultes), mais aussi quelque parent éloigné ou voisin, y contribue. Le souk est un des hauts lieux de déploiement de ces solidarités. Cette participation du mari aux activités de sa femme 
montre bien la redéfinition des rôles qui s'opère, par la migration, dans la sphère productive. Le pouvoir migratoire acquis par la femme lui confère, en ce qui concerne l'entreprise, un pouvoir de décision bien supérieur à celui de son mari. Le mari devient son assistant, tandis que la femme est le véritable chef d'entreprise. Aussi, si la participation du mari conditionne bien la réussite, les femmes rappellent, dans l'intimité, qu'elles détiennent le destin de l'entreprise et de leur famille entre leurs mains, même si tous tirent profit des bénéfices dégagés.

« Si on avait attendu mon père pour s'enrichir, on coucherait encore avec les poules » (Sabrina, 25 ans).

«Les hommes n'ont pas de goût, ils achètent n'importe quoi. Mon mari c'est un joueur, il aime jouer, regarde la nouvelle voiture qu'il a achetée, un vrai gadget ! C'est moi qui retrousse mes manches » (Lamia, 37 ans).

\section{De bonnes mères/filles de famille}

Si la migration donne lieu à une redéfinition des rôles dans la sphère productive, en revanche, les changements sont moins nets dans la sphère reproductive, où la femme continue d'assurer les rôles de mère de famille et de parfaite épouse, ou de jeune fille de maison, pour celles qui suivent leur mère et vivent encore chez leurs parents.

De nombreux travaux, dans des contextes géographiques différents, ont montré que le type de migration choisi est lié à une appartenance de genre. À cet égard, la circulation migratoire, à la différence d'une migration d'installation, permet plus facilement aux femmes de maintenir leurs responsabilités reproductives (Ellis, Conway et Bailey, 1996 ; Morokvasic, 1996, 2003 ; Salaff, 1997). Comme elles le précisent à plusieurs reprises, les commerçantes tunisiennes choisissent la circulation pour ne pas avoir à effectuer une migration d'installation en Europe. Quelques-unes d'entre elles ont même une expérience antérieure de permanence en Italie, mais ont préféré se réinstaller au pays, soit parce que les conditions de vie en Italie, caractérisées par la précarisation croissante du statut des migrants, étaient jugées insatisfaisantes, soit parce qu'elles étaient dans l'impossibilité de faire venir leur famille. La circulation est vécue comme une stratégie particulièrement efficace, dans la mesure où elle permet d'améliorer leur statut et leur niveau de vie tout en n'abandonnant pas leurs responsabilités dans la sphère reproductive. Cette double présence, sur les routes du commerce et au foyer, demande beaucoup d'investissement de la part des femmes, qui doivent toujours être ici et là-bas, à la maison et en voyage à la fois.

«J'ai un rythme de vie très dur. Quand je rentre de voyage, je me lève très tôt le matin, je fais le ménage, surtout dans cette maison. Parce que cette maison est la principale, les autres sont plus petites, plus faciles à nettoyer. Après quand je termine ce travail à dix heures du matin, j'ai les clients qui téléphonent et qui viennent jusqu'à une heure du matin! Surtout quand il y a un nouvel arrivage. Tu peux pas imaginer, je reste trois jours sans rien manger » (Zahra).

31 En même temps, pratiquer une migration circulaire réduit notablement le coût social et familial que comporterait le fait de négliger son foyer. Elle donne une légitimité à la femme, qui est, par conséquent, valorisée. En ce sens, cette présence au foyer permet de désamorcer les représentations négatives couramment associées aux circulantes. Ainsi, l'adjectif le plus souvent utilisé pour les qualifier dans leur entourage est le «courage». Dans les entretiens, l'accent est mis sur le fait que ces femmes n'oublient jamais leur famille.Il faut alors trouver des solutions pour mener à la fois son activité de femme au foyer et celle d'entrepreneuse. Certains travaux sont délégués à des 
voisines, des belles-sœurs (le soutien de la famille élargie est ici essentiel), ou à des petites bonnes, pour les plus fortunées d'entre elles. Cependant, si les femmes délèguent une partie de leurs responsabilités productives, elles le font de façon limitée et maintiennent toujours un contrôle sur la sphère domestique : on pourrait dire, en reprenant le vocabulaire entrepreneurial, qu'elles sous-traitent une partie de leurs activités, mais demeurent les seules donneuses d'ordre. Une autre médiation consiste à emporter ses enfants "sous le bras", en voyage, notamment lorsqu'ils sont en bas âge. Hafida, la fille de Hayet, âgée de 23 ans, raconte :

«Moi et mes frères, on a tous vécu l'expérience de l'Italie Ma mère était enceinte de plus de deux mois, de la petite : elle était encore sur les routes. Parce qu'on peut pas rester... on a une amie elle était au neuvième mois de grossesse, elle est allée en Italie, elle a ramené tout, parce qu'elle sait qu'elle va rester pendant 40 jours ou deux mois sans rien faire alors elle doit se préparer convenablement alors elle a fait le voyage en double pour avoir les économies de la grossesse et du nouveau-né ».

Ainsi, la réussite de l'entreprise féminine se base sur l'équilibre du couple et de la famille, et la femme conserve une grande partie de ses responsabilités dans la sphère reproductive. La participation et l'aval du mari sur les activités féminines sont essentiels. On peut rejoindre à cet égard les analyses de D. Vaiou et K. Simonsen qui voient le foyer comme un lieu de négociation, d'expression de points de vue, d'intérêts et de stratégies multiples. Pour les commerçantes circulantes, les changements dans les relations de genre se font sur la base d'arrangements constants, d'adaptations progressives plutôt que de transformations radicales (Simonsen et Vaiou, 1996). D'un point de vue méthodologique, cela montre bien qu'il est nécessaire de tenir compte à la fois des changements introduits dans la sphère productive et reproductive, pour comprendre les motivations et les choix de migration (Zlotnik, 1995; Zontini, 2002 ; Kofman, 2003).

Cette négociation des rôles productifs et reproductifs au sein de la cellule familiale s'assortit d'autres ajustements. Sur les routes commerciales et les places d'achat, les femmes sont confrontées à différents obstacles : négociation d'un passage en douane, marchandage avec les grossistes... à ces difficultés, qui ne sont pas propres aux femmes, s'ajoutent celles liées au fait de traverser des espaces essentiellement masculins, sur lesquels les femmes ont peu de légitimité. Sur quelles ressources peuvent-elles s'appuyer pour traverser les frontières matérielles et symboliques des espaces de la circulation commerciale?

\section{Des « savoir-circuler » spécifiquement féminins}

\section{De l'usage du réseau de " copines » : entre mise en commun des ressources et négociation de la mixité}

Les commerçantes mettent en œuvre des modes d'organisation originaux basés sur des compétences et des solidarités féminines. Tout d'abord, elles ne voyagent que très rarement seules et se déplacent généralement en «grappes» (Boubakri, 2003) de trois à six personnes qu'elles appellent des « copines ». Les compagnes de voyage varient d'une expédition à l'autre, mais sont toujours sélectionnées au sein d'un groupe fixe d'une cinquantaine de personnes autour duquel gravitent quelques dizaines d'«électrons libres ", c'est-à-dire d'amies et de connaissances qui occasionnellement se greffent sur 
le noyau dur pour faire quelques courses à Naples ${ }^{8}$. Cette spécialité peut se transmettre entre sœurs ou de mère en fille. Sabrina, fille de commerçante, raconte :

"Avant, quand j'étais une élève, quand j'avais des cours et que je ne pouvais pas voyager, je laissais de l'argent à ma mère. Je lui disais: "ramène-moi tel ou tel objet”. Dès les premiers jours, il ne restait plus rien chez moi. Je vendais au lycée, à mes copines les petits trucs, les pinces pour les cheveux, alors cette passion a commencé à grandir en moi et j'ai voulu la suivre ».

Les réseaux de commerçantes reproduisent en partie les formes de sociabilité traditionnelles de la ville maghrébine, dans laquelle ce sont avant tout les liens familiaux, de quartier, ainsi qu'une origine régionale commune qui orientent les territorialités féminines (Berry-Chikhaoui, 2000). Cependant, d'autres formes de rencontre apparaissent, et contribuent à diversifier les réseaux de relation des commerçantes. La fréquentation commune des marchés tunisiens, tout comme celle des routes commerciales et des places d'achat sont autant d'occasions de nouer des connaissances. De nouveaux réseaux de sociabilité se créent, qui permettent une autonomisation par rapport aux structures sociales traditionnelles. Cet aspect du métier est perçu par les femmes comme un de ses principaux avantages.

L'entrée dans le groupe des femmes circulantes s'effectue par une sorte de cooptation. Elle nécessite que celles-ci aient été introduites, «élues» en tant que personnes de confiance par l'une d'entre elles. Les solidarités nouées au cours des déplacements, qui sont plus ou moins fortes, peuvent se solder par un mariage. Plusieurs femmes ont ainsi marié leurs plus jeunes compagnes de voyage à leur fils, ce qui prouve bien que les voyageuses ne sont pas considérées, du moins dans le monde des circulantes, comme de mauvais partis.

L'entraide a toutefois ses limites. Seuls les savoir-circuler sont mis en commun, tandis que les entreprises demeurent individuelles. Les bénéfices dégagés tout comme les capitaux engagés ne sont guère partagés. Le capital financier de départ peut être acquis en recourant à un prêt auprès d'une autre femme, mais celle-ci fait rarement partie du groupe des circulantes. Par ailleurs, les conflits ne sont guères absents, ni les formes de concurrence dont les femmes se plaignent toujours plus avec la diffusion du commerce transfrontalier. Il n'est pas rare que l'une d'entre elles aille jusqu'à soudoyer des grossistes basés à Naples pour savoir quel type de produit sa collègue a acheté. Plus qu'une entreprise commune, le groupe de copines serait donc une sorte de club, de groupement d'intérêts : à l'intérieur du groupe, il n'est pas rare que l'on change de partenaire, même si certaines sont préférées à d'autres, en général parce qu'elles sont plus expérimentées. Le choix de la compagne de voyage se fait également selon la disponibilité des unes et des autres à partir, c'est-à-dire le fait qu'elles se soient reconstituées un capital d'achat (qu'elles aient donc écoulé une partie de leur stock), qu'elles aient pu obtenir un visa ${ }^{9} . .$.

38 Le voyage en groupe comporte pourtant certains désavantages, à savoir une visibilité externe plus importante, mais aussi une visibilité dans le groupe, qui est à l'origine d'une tendance à acheter les mêmes types de produits. Cependant, malgré ses désavantages, il est préféré pour plusieurs raisons. Tout d'abord, il permet de mettre en commun des ressources immatérielles comme la connaissance des routes et des personnes, et éventuellement de la langue italienne. Ainsi, l'initiation au voyage d'une nouvelle consiste à lui «montrer la route » au double sens pratique et métaphorique de l'expression, c'est-à-dire à lui transmettre les chemins à emprunter et les personnes à 
connaître, qui sont la base des compétences circulatoires de ces femmes. En outre, il est plus agréable de voyager en compagnie : dans les chambres d'hôtel et dans les lieux de circulation se créent parfois de véritables ambiances de fêtes qui permettent de rendre supportable, et même plaisante, une vie dans la mobilité. Le voyage en groupe a également des avantages économiques, puisqu'il permet de réduire les coûts : chambres d'hôtel et cabines de bateaux, locations de camionnettes et frais variés sont partagés. Mais surtout la présence du groupe permet d'être plus préparée à affronter la société locale et surtout de limiter la probabilité de faire de mauvaises rencontres. Les femmes qui voyagent en groupe n'ont pas vécu en Italie et connaissent mal le pays, en dehors des espaces fréquentés pendant les voyages. Ce type de voyage réduit également le coût affectif de la migration pour les hommes qui restent en Tunisie puisque le réseau de " copines » exerce une fonction d'encadrement et de contrôle sur le comportement des femmes. Cela montre bien que la migration ne revient pas à se soustraire totalement au contrôle masculin. Le voyage en groupe permet, en quelque sorte, de rassurer ceux qui demeurent au pays, maris et enfants. Il a, de ce point de vue, une fonction d'« annulation de la mixité » (Navez-Bouchanine, 1997 ; Berry-Chikhaoui, 2000).

Ainsi, l'observation de l'organisation en réseaux de ces femmes permet de dépasser une vision trop consensuelle et figée des solidarités (Riccio, 2000). Plus qu'une base ethnique, ce sont bien des expériences et des objectifs partagés, la pratique et le goût du commerce en l'occurrence, ainsi que des exigences de contrôle social, qui lient ces femmes (Morokvasic, 1999, 2003 ; Péraldi, 2001 ; Hily et Rinaudo, 2003). Il n'existe pas de contradiction, malgré les apparences, entre le fait que ces femmes s'appuient sur une structure communautaire pour voyager mais, en même temps, se livrent une forte concurrence entre elles. Ce caractère ambivalent des réseaux a été mis en lumière par une équipe de recherche portugaise qui a travaillé sur la circulation commerciale des Cap-verdiennes, les célèbres "rebidantes $»^{10}$ (Marques, Santos et Araujo, 2001). Ces auteurs montrent que la compétitivité économique et la construction active de réseaux sociaux constituent en réalité les deux faces de la même pièce, et proposent de parler à ce sujet d' " autonomie encastrée » des commerçantes : «ce qui émerge des témoignages des rebidantes, c'est précisément la recherche d'un équilibre entre ouverture et fermeture, un compromis entre nécessités sociales et opportunités commerciales, entre association et séparation 》 (2001: 292).

Parmi les femmes interrogées, une minorité, parce qu'elle dispose d'autres ressources, se démarque du groupe et préfère voyager seule. Moins captives, ces femmes ont pour point commun d'avoir connu une expérience migratoire sur le long terme, qui leur permet de gérer par elles-mêmes certaines situations. Peu nombreuses, elles se détachent du profil commun, car elles disposent d'un réseau de relations en Italie pour effectuer leurs démarches. Ce sont celles qui ont connu les réussites les plus éclatantes. Parmi ces femmes, Lamia qui, avant de s'installer de nouveau en Tunisie a vécu plusieurs années en Italie avec sa famille, est la plus radicale: possédant deux boutiques destinées à la classe sociale la plus favorisée de la ville, elle déclare préférer payer ses voyages au prix fort plutôt que d'avoir à se déplacer avec les autres femmes à Naples.

\section{La féminité comme tactique de traversée des espaces}

41 Au-delà du voyage en groupe, il existe des ruses proprement féminines de négociation de la mobilité, qui participent de la prise de «pouvoir migratoire» de ces femmes. Ces 
ruses consistent à jouer de sa position de femme pour contourner des contraintes, comme celles liées à l'action des dispositifs légaux qui devraient empêcher l'exercice de leur activité. "Je mets mes petites culottes au-dessus de ma valise: qui ose fouiller qu'il fouille!» déclare ainsi Sabrina, qui compte sur cette ruse pour provoquer la gêne et détourner le regard des douaniers sur ses achats. Pour attirer la compassion d'un douanier ou pour faire baisser les prix d'un grossiste, on n'hésite pas à insister sur sa faiblesse et son âge avancé, tandis que le port du hijab, qui n'est pratiqué que dans certaines situations, est censé forcer la distance (pour les Italiens) et le respect (dans le cas des Maghrébins) des interlocuteurs masculins. Cet usage tactique du port du voile par les femmes a été étudié par Barbara Cooper ${ }^{11}$ qui a mis en évidence la sensation de sécurité associée à cet usage, ainsi que la mobilité qu'il permet. Étudiant les pratiques spatiales des femmes de Maradi au Niger, B. Cooper voit dans le port du voile un "moyen critique pour franchir les limites d'un espace domestique ", et propose de parler à ce sujet de "mobilité tactique» : « il me semble crucial que nous commencions à tenir compte de cette sorte de mobilité tactique, mobilité à laquelle les femmes participent sans ostentation et qui modifie profondément les options qui leur sont accessibles ", écrit-elle (Cooper, 2001 : 93).

Le mode vestimentaire, l'instrumentalisation du stigmate qu'est le voile, devient alors l'outil de «l'art du faible », du plus démuni (de Certeau, 2002). Le marqueur identitaire devient l'instrument d'une ruse ou "tactique» de contournement des dispositifs juridiques et étatiques, au sens que M. de Certeau lui accorde. Dans un lieu et à une époque où la situation est défavorable à ces femmes, elles vont chercher à la tourner à leur avantage ${ }^{12}$. En outre, de même qu'il existe des stratégies féminines de négociation de la mobilité, les femmes s'approprient de façon spécifique les espaces traversés, comme permet de l'illustrer l'exemple napolitain.

\section{Territorialités féminines sur les places d'achat : des pratiques genrées des espaces}

\section{Le dispositif napolitain}

43 Le développement des circulations commerciales soulève la question de la relation qui s'établit entre les circulants et les espaces qu'ils traversent. Alain Tarrius constate, à travers la notion de "territoire circulatoire», la capacité des petits migrants, entrepreneurs de la « mondialisation par le bas ", à donner sens à des lieux distants, et les oppose aux cadres circulants internationaux, "sous produit des stratégies multinationales des firmes", incapables de produire "des identités spécifiques, ni des traces territorialisées d'un type nouveau »(1996: 102). Les «territoires circulatoires» des nomades, qu'il définit comme "socialisation d'espaces-supports à des pratiques de mobilité", se superposent à ceux des sédentaires et ne correspondent pas aux mêmes logiques, ni aux mêmes hiérarchies. Cette notion permet ainsi de donner une cohérence à l'ensemble discontinu des espaces agrégés et transformés par les pratiques des circulants, en montrant que des lieux peuvent être appropriés et investis de signification sans qu'il y ait nécessairement présence permanente d'un groupe dans un espace donné.

Dans l'aire métropolitaine de Naples, un territoire commercial spécifique s'est structuré de manière progressive, au fur et à mesure que les flux de circulants se renforçaient ${ }^{13}$. Un dispositif mouvant, constitué des différents lieux d'achat dans l'agglomération, mais aussi des lieux de captage et de redistribution des flux d'hommes 
et de marchandises (aéroport, port, plateforme multimodale), est apparu. Le quartier de la gare de Naples, en tant que carrefour d'opportunités (croisement de réseaux de transports, lieu d'achat en gros, principal lieu de concentration des hôtels et des restaurants communautaires dans la ville), est devenu une place centrale au sein de ce dispositif.

La mise en relation du quartier de la gare avec les différents lieux du dispositif est assurée par des intermédiaires, individus dont les compétences commerciales et circulatoires sont particulièrement affirmées. Figures logistiques et de mise en contact de la place marchande, ils se chargent d'acheminer les circulants vers les lieux d'achat et de les mettre en relation avec leurs interlocuteurs commerciaux (grossistes, transitaires, hôteliers...). Le recours à ces intermédiaires, qui font parfois figure de traducteur, mais surtout d'interface et de garant dans un contexte où les échanges se basent essentiellement sur la réputation et le face-à-face, est rendu nécessaire par le caractère informel de la plupart des transactions. Ils organisent la rencontre entre sédentaires et circulants. Loin de se cantonner à un rôle économique, ils jouent alors un rôle de "médiation culturelle " particulièrement important en cherchant à construire auprès des sédentaires les conditions de l'accueil des circulants (Ma Mung, 1992).

De même que la circulation commerciale ne revêt pas la même signification pour les hommes que pour les femmes, leurs pratiques et appropriations spatiales au sein du dispositif napolitain sont modelées par leur appartenance de genre.

\section{Des appropriations spécifiques, centrées sur les espaces privés}

Dans le quartier de la gare de Naples, il est possible d'opposer, schématiquement, les espaces publics (cafés, trottoirs), fréquentés par les hommes, et les espaces privés, appropriés par les femmes circulantes ${ }^{14}$. Les circulantes se sentent mal à l'aise dans ce quartier d'immigration masculine fortement stigmatisé. Elles craignent d'être assimilées aux femmes maghrébines du quartier, qu'elles considèrent comme des femmes de mauvaises mœurs. En effet, les seules femmes maghrébines qu'on retrouve sur les trottoirs du quartier sont des prostituées installées à Naples, qui correspondent alors davantage à la figure de la primo-migrante évoquée au début de ce texte. C'est pourquoi les commerçantes ne fréquentent les espaces publics qu'en cas de nécessité, à l'occasion de leurs achats, et opèrent une sorte de repli sur les espaces clos des hôtels.

La fréquence de leurs déplacements à Naples, leur caractère routinier, leur confère un pouvoir contractuel et de transformation de ces lieux. Certains propriétaires d'hôtel ont ainsi revu l'organisation de leur établissement en fonction des souhaits de cette clientèle exigeante. Des chambres ont été aménagées en mini-appartements car les femmes refusent de dîner dans les restaurants du quartier et préfèrent préparer leur souper à l'hôtel. Les femmes commerçantes se sont approprié ces espaces: elles en parlent en disant "chez nous", s'invitent à y prendre le thé et à y déguster les mets qu'elles apportent de Tunisie. Sorte d'espace domestique bis, elles y ont reconstruit une intimité. C'est parfois un véritable attachement à ces lieux qui se produit, peu compréhensible par ceux qui ne participent pas à la circulation commerciale :

«À dire vrai, je me suis attachée à cet hôtel. Ils sont très disponibles, et puis c'est une gestion familiale, tranquille. Ils ne me font aucun problème quand j'apporte mes bagages et tout le reste. Ma fille ne me comprend pas, elle dit que cet hôtel au lieu de s'appeler "Aurore" devrait s'appeler "hôtel Horreur" ! (Loubna). 
De récentes observations montrent que la perception et la pratique de l'espace ne sont pas les mêmes pour toutes les femmes. L'appartenance de genre ne suffit pas à déterminer des pratiques et représentations spatiales, mais doit être mise en relation avec d'autres variables : génération, activité, régime de mobilité...

\section{Des individus qui permettent la traversée des espaces masculins : les intermédiaires}

Les relations des femmes avec l'espace public s'effectuent à travers le filtrage des intermédiaires commerciaux, qui se chargent de les prémunir des désagréments venus du monde masculin. En effet, les femmes sont parfois très inquiètes de la menace potentielle que représentent les Italiens, qu'elles jugent beaucoup trop zélés en courtoisie. Aussi, bien plus qu'une simple figure logistique et de mise en relation de l'offre et de la demande commerciale, ces intermédiaires sont pour les femmes de véritables gardiens du bon déroulement des événements. Leurs compétences, loin de se limiter aux échanges marchands, s'étendent à l'ensemble de leurs pratiques quotidiennes à Naples :

«Hier, les femmes m'ont appelé à trois heures du matin. Elles me disent: "viens vite Ahmed!". Je les trouve sur le trottoir, en bas de l'hôtel, en chemise de nuit: elles m'expliquent qu'un vagabond, un ivrogne, est rentré dans les couloirs pendant la nuit, elles veulent plus rester à l'hôtel, elles ont trop peur, moi j'ai dû garder la chambre après, toute la nuit. Mais le patron de l'hôtel, il va m'entendre, il a intérêt à faire quelque chose parce qu'il nous verra plus si ça continue...» (Ahmed, intermédiaire tunisien).

51 Cette anecdote fait émerger une situation de crise, liée à l'irruption dans l'intimité des femmes d'un personnage " $d u$ dehors». L'intermédiaire cherche à neutraliser cette situation en faisant comprendre au propriétaire qu'il doit surveiller le passage dans son hôtel s'il souhaite conserver cette clientèle féminine. De ce point de vue, il est un garant moral. Les enjeux liés à son rôle semblent d'autant plus importants que Naples se situe hors du dar al islam, comme le soulignent nombre de nos interlocutrices féminines ${ }^{15}$. Pour ces femmes, c'est également parce que les interlocuteurs sédentaires sont essentiellement des Italiens, en d'autres termes des non-musulmans, que la présence des intermédiaires commerciaux est importante. Elles expliquent, par exemple, ne pas avoir recours aux mêmes types de services lors de leurs déplacements à Istanbul, où leurs interlocuteurs sont musulmans et parfois même arabophones ${ }^{16}$. L'intermédiaire devient une sorte de passeur, qui permet la traversée de frontières symboliques. Il est une figure centrale de l'éventail relationnel de ces femmes, dans la mesure où il les met en relation avec des univers commerciaux différents tout en cherchant à les prémunir de certaines rencontres.

\section{Conclusion}

Par leurs capacités à s'inscrire entre ici et là-bas, à développer des champs relationnels et des «routines » qui dépassent le cadre de leur État d'origine, les commerçantes tunisiennes apparaissent clairement comme des transmigrantes. Cependant, comme l'ont mis en évidence des approches critiques aux phénomènes de transnationalisme, une perspective de genre implique de tenir compte de deux aspects, que l'on a cherché 
à mettre en lumière (Morokvasic, 2003 ; Pratt, Yeoh, 2003) : tout d'abord, la circulation est un phénomène sexuellement différencié, ce qui a des conséquences sur l'organisation et sur l'usage des espaces des circulants. Ensuite, la transmigration n'est pas nécessairement un phénomène émancipateur, transgressif et générateur de mobilité sociale. Dans le cas des commerçantes tunisiennes, la circulation donne lieu à des formes d'autonomisation, de reformulation des statuts et de mobilité sociale, mais celle-ci est négociée, à la fois au sein du foyer et dans les espaces traversés. Par ailleurs, si l'on peut se réjouir de la participation croissante des femmes maghrébines aux sphères publique et professionnelle, il convient de remarquer que la circulation commerciale est également le produit de contraintes structurelles ; son développement est lié à la fragilisation des statuts des migrants dans les pays d'immigration, ainsi qu'aux difficultés rencontrées par les classes moyennes des pays en transition.

Aussi, c'est selon la multiplicité de leurs activités et de leurs positions sociales qu'il convient d'envisager les trajectoires, carrières et itinéraires de ces femmes. Elles sont tout à la fois tunisiennes, maghrébines et musulmanes ; migrantes et femmes au foyer ; mères ou filles, épouses, "copines », commerçantes et passeuses. Leurs parcours et leurs modes d'organisation reflètent les évolutions et les blocages socio-économiques, les pratiques culturelles et de consommation des sociétés qu'elles traversent. Ils reflètent "les positions des hommes et des femmes dans la société" (Bjeren, 1997), en même temps qu'ils les transforment. De ce point de vue, si « la migration féminine transgresse le modèle social traditionnel de la femme " (Ramirez, 1999), il convient de constater qu'elle le fait en douceur, dans la continuité, entrant en contradiction avec certaines analyses qui voient dans la mobilité féminine l'expression d'un déracinement ou du moins d'une rupture avec les liens précédents l'émigration. En effet, ces migrantes ne sont pas des marginales, mais bien au cœur de dynamiques qui concernent des États, dans lesquels les petits, les «fourmis », pour reprendre la métaphore d'Alain Tarrius, développent des modes d'organisation informels et alternatifs permettant d'appuyer leurs stratégies de promotion (Tarrius, 1992 ; Péraldi, 2001).

54 Certes, les résultats sont variables selon les femmes et sont, dans tous les cas, limités. Cependant, comme elles le soulignent à maintes reprises, les femmes, à travers la circulation, ne paient pas le coût social élevé d'une migration définitive, avec les formes de rupture qu'elle pourrait comporter, tandis que l'organisation en groupe permet de limiter l'impact du voyage. Tirant profit en de multiples occasions de leur avantage migratoire, manipulant leur féminité et leurs stigmates, se créant un large éventail relationnel, ces femmes sont parvenues à tirer profit, tout en les transformant, d'espaces distants.

\section{BIBLIOGRAPHIE}

ANTHIAS Floya, LAZARIDIS Gabriella Éds (2000) Gender and Migration in Southern Europe. Women on the move, Oxford, Berg, $263 \mathrm{p}$. 
ASLAFY-GAUTHIER Catherine (2002) Les routes du bled : commerce et mobilités marocaines, in Michel Péraldi Éd., La fin des norias ? Réseaux migrants dans les économies marchandes en Méditerranée, Paris, Maisonneuve et Larose, pp. 193-216.

BATTEGAY Alain (2003) Les recompositions d'une centralité commerçante immigrée : la Place du Pont à Lyon, Revue Européenne des Migrations Internationales, 19 (2), pp. 9-20.

BEKKAR Radia (2004) Femmes du Maghreb dans la recherche en sciences sociales, Maghreb Machrek, 179, pp. 13-28.

BERRY-CHIKHAOUI Isabelle (2000) Territorialités féminines dans la ville au Maghreb : quelques éléments de réflexion, inChristian Lochon, Véronique Bodin, Jean-Pierre Doumenge Éds., Femmes et Islam, Actes du colloque « Rôle et statut des femmes dans les sociétés contemporaines de tradition musulmane », Paris, CHEAM, 15-16 décembre 1999, pp. 114-126.

BJEREN Gunilla (1997) « Gender and Reproduction », in Thomas Hammar, Grete Brochmann, Kristof Tamas, Thomas Faist Eds., International Migration, Immobility and Development, pp. 219-246.

BOUBAKRI Hassen (2003) La circulation des Tunisiens en Italie et en France, in Marie-Antoinette Hily, Emmanuel Ma Mung Éds., Catégories et lieux des circulations migratoires, Rapport de recherche pour la MIRE, Ministère du travail, convention n ${ }^{\circ}$ WKO 324, pp. 48-62.

BOULAHBEL Yeza (1996) Dames de France et d'Algérie : une stratégie d'autonomisation à l'ombre des traditions, Cahiers du Mage, 3, pp. 105-114.

BREDELOUP Sylvie (2001) Marseille. Carrefour des mobilités africaines in Michel Péraldi Éd., Cabas et containers, Activités marchandes informelles et réseaux migrants transfrontaliers, Paris, Maisonneuve et Larose, pp. 65-105.

CAMPANI Giovanna (1995), Women Migrants : from marginal subjects to social actors, in Robin Cohen Ed., The Cambridge Survey of World Migration, Cambridge, Cambridge University Press, pp. 546-550.

CERTEAU Michel de (2002) L'invention du quotidien. Arts de faire, col. Folio Essais, Paris, Gallimard, 350 p. [prem. éd. 1980].

CHAPONNIÈRE Jean Raphaël (2002) Le tourisme, enjeu en Méditerranée, Fiche de Synthèse de la Direction des Relations Economiques Extérieures, 7 p.

CHARRAD Mounira (1998) Cultural Diversity Within Islam : Veils and Laws in Tunisia, in Herbert L. Bodman, Nayereh Tohidi Eds, Women in Muslim Societies, Diversity Within Unity, Londres, Rienner, pp. 63-79.

COOPER Barbara (1997) « Gender, Movement and History : Social and Spatial Transformations in 20th century Maradi Niger ", Environment and Planning D : Society and Space, 15, pp.195-221, trad. Française, « Le Genre sexuel, le mouvement et l'histoire : transformations sociales et spatiales au $\mathrm{xx}^{\mathrm{e}}$ siècle à Maradi au Niger ", Stazsack J. F et al., 2001, Géographies Anglo-saxonnes, Paris, Belin, pp. 80-94.

DELI Fedime (2002) La maîtrise du commerce international du textile par les patrons arabes de Mardîn à Laleli (Istanbul) in Michel Péraldi Éd., La fin des norias ?, Paris, Maisonneuve et Larose, pp. 287-305.

ELLIS Mark, CONWAY Dennis, BAILEY Adrian J. (1996) The circular migration of Puerto Rican Women : towards a gendered explanation, International Migration, 34 (1), pp. 31-64.

EL HARIRI Saâdia (2003) Les Marocaines au cœur d'un nouveau circuit d'échanges marchands : entre ici et là-bas, Revue Européenne des Migrations Internationales, 19 (1), pp. 195-222. 
GOFFMAN Erving (1998) Les rites d'interaction, Paris, Les Éditions de Minuit, 231 p. [prem. éd. 1974].

GRANOVETTER Mark (2000) Le marché autrement, essais de Mark Granovetter, col. Sociologie Économique, Paris, Desclée de Brouwer, 239 p.

HILY Marie-Antoinette, RINAUDO Christian (2003) Cosmopolitisme et altérité, les nouveaux migrants dans l'économie informelle, Tsantsa, 8, pp. 48-57.

HUMARAU Béatrice (1997) Grand commerce féminin, hiérarchies et solidarités en Afrique de l'Ouest, Politique africaine, 67, pp. 89-102.

JOMNI Sofia (2000) Les femmes dans le secteur informel en Tunisie : dualité des formes d'activité et reconnaissance sociale du travail féminin, inChristian Lochon, Véronique Bodin, Jean-Pierre Doumenge Éds., Femmes et Islam, Actes du colloque « Rôle et statut des femmes dans les sociétés contemporaines de tradition musulmane », Paris, CHEAM, 15-16 décembre 1999, pp. 128-155.

KAPCHAN Deborah (1996) Gender on the market, Philadelphie, University of Pensylvania Press, 325 p.

KOFMAN Eleonore (2003) Gender and International Migration : a Critique of Theoretical Reductionism, texte présenté à la $44^{\text {ème }}$ convention de l'International Studies Association, Portland, 25/02-01/03.

LAMINE Rhida (2000) Villes et citadins du Sahel Central, Tunis, L'or du temps, 577 p.

MARQUES Margarida, SANTOS, Rui, ARAUJO Fernando (2001) Ariadne's Thread. Cape Verdean Women in Transnational Webs, Global Networks, 1 (3), pp. 283-306.

MASSEY Doreen, MC DOWELL Linda (1994) A woman's place ?, in Doreen Massey Ed., Space, place and gender, Minneapolis, University of Minesota Press, pp. 191-211.

MASSEY Douglas (2002) La ricerca sulle migrazioni nel XXI secolo, in Asher Colombo, Giuseppe Sciortino Eds., Stranieri in Italia, Bologne, Il Mulino, pp. 25-52.

MA MUNG Emmanuel (1992) Commerce ethnique et médiation culturelle, Migrations Société, 22-23 (4), pp. 87-90.

MC DOWELL Linda (1999) City Life and Difference : Negociating Diversity, in John Allen, Doreen Massey, Michael Pryke Eds., Unsettling cities, Londres, Routledge, pp. 95-135.

MC LEOD Arlene (1991) Accomodating Protest. Working Women and the New Veiling inCairo, New York, Columbia University Press, $206 \mathrm{p}$.

MERNISSI Fatima (2001) Le Harem et l'Occident, Paris, Albin Michel, 230 p.

MISSAOUI Lamia (1995) La généralisation du commerce transfrontalier : petit ici, notable là-bas, Revue Européenne des Migrations Internationales, 11 (1), pp. 53-75.

MOGHADAM Valentine (1998) Women, Work and Economic Reform in the Middle East and North Africa, Londres, Lynne Rienner Publishers, 259 p.

MOORE Henrietta L. (1994) A passion for difference. Essays in Anthropology and Gender, Cambridge, Polity Press, 177 p.

MOROKVASIC Mirjana (1996), Entre l'Est et l'Ouest, des migrations pendulaires, in Mirjana Morokvasic, Rudoph Hedwig Éds., Migrants. Les nouvelles mobilités en Europe, Paris, L'Harmattan, pp. 119-157. 
MOROKVASIC Mirjana (1999) La mobilité transnationale comme ressource : le cas des migrants d'Europe de l'Est, Cultures et Conflits, 42-43, pp. 105-122.

MOROKVASIC Mirjana (2003) Transnational mobility and gender : a view from post-wall Europe, in Mirjana Morokvasic, Umut Erel, Kyoko Shinozaki Eds, Crossing Borders and Shifting Boundaries, Gender on the move, Opladen, Leske et Budrich, pp. 101-133.

MOZERE Liane (2002) Les domestiques philippines « entrepreneures d'elles-mêmes ». Le marché mondial de la domesticité, Paris, Rapport pour la mission du patrimoine ethnologique, $199 \mathrm{p}$.

NAVEZ-BOUCHANINE Françoise (1997) Habiter la ville marocaine, Paris, L'Harmattan, 315 p.

PERALDI Michel Éd. (2001) Cabas et containers, Activités marchandes informelles et réseaux migrants transfrontaliers, Paris, Maisonneuve et Larose, $361 \mathrm{p}$.

PERALDI Michel (2005) (sous presse) Routes Algériennes, in Anteby Lisa, Berthomière William, Sheffer Gabriel Éds., 2000 ans de Diasporas, Rennes, Presses Universitaires de Rennes.

PRATT Geraldine, YEOH Brenda (2003) Transnational (counter-) topographies, Gender, Place and culture, 10 (2), pp. 159-166.

RAMIREZ Angeles (1999) La valeur du travail, l'insertion dans le marché du travail des immigrées marocaines en Espagne, Revue Européenne des Migrations Internationales, 15 (2), pp. 9-36.

RAMIREZ Angeles (2002) Les migrations internationales et les rapports de sexe, les femmes marocaines en Espagne, in Mohamed Charef Éd., Les migrations au féminin, Agadir, Sud contact, pp. 85-98.

RICCIO Bruno (2000) Pregi e limiti dell' approccio transnazionale al fenomeno migratorio, Etnoantropologia, 8-9, pp. 253-262.

SALAFF Janet W. (1997) The gendered Social Organization of Migration as Work, Asian and Pacific Migration Journal, 6 (3-4), pp. 295-316.

SCHMOLL Camille (2001) Immigration et nouvelles marges productives dans l'aire métropolitaine de Naples, Bulletin de l'Association des Géographes Français, 4, pp. 403-413.

SECOR Anna J. (2002) The veil and urban space in Istanbul : women's dress, mobility and islamic knowledge, Gender, Place and Culture, 9 (1) pp. 5-22.

SENGEL Marie (2000) Nana-Benz de Noailles, Hommes et Migrations, 1224, pp. 71-78.

SILVEY Rachel (2004) Power, difference and mobility : feminist advances in migration studies, Progress in Human Geography, 28 (4), pp. 490-506.

SIMON Gildas (1990) Les diasporas maghrébines et la construction européenne, Revue Européenne des Migrations Internationales, 6 (2), pp. 97-106.

SIMONSEN Kirsten, VAIOU Dina (1996) Women's Lives and the Making of the city, Experiences from North and South of Europe, International Journal of Urban and Regional Research, 20 (3), pp. 446-465.

TARRIUS Alain (1992) Les Fourmis d'Europe. Migrants riches, migrants pauvres et nouvelles villes internationales, Paris, L'Harmattan, 207 p.

TARRIUS Alain (1996) Territoires circulatoires et espaces urbains, in Mirjana Morokvasic, Rudolph Hedwig Éds, Migrants. Les nouvelles mobilités en Europe, Paris, L'Harmattan, pp. 93-117.

TROIN Jean-François (1975) Les souks marocains : marchés ruraux et organisation de l'espace dans la moitié nord du Maroc, Aix-en-Provence, Edisud, 503 p. 
ZLOTNIK Hania (1995) Migration and the Family : the Female Perspective, Asian and Pacific

Migration Journal, 42, 2-3, pp. 253-271.

ZONTINI Elisabetta (2002) Towards a Comparative Study of Female Migrants in Southern Europe, Studi Emigrazione, 145, pp. 107-134.

\section{NOTES}

1. À Sousse, selon les témoignages recueillis, environ une cinquantaine de femmes pratiquerait le commerce transnational vers l'Italie de façon régulière, mais les acheteuses occasionnelles seraient bien plus nombreuses (plusieurs centaines). L'évaluation du phénomène demeure très délicate, notamment du fait de la dispersion des itinéraires (voies aérienne et maritime, multiplicité des lieux d'entrée en Italie), et de la diversité des statuts légaux des commerçantes (détentrices de permis de séjour ou de visas de tourisme, titulaires d'une licence d'import-export ou d'un simple permis de vente au marché...).

2. Ce texte se base sur une enquête ethnographique réalisée entre janvier 2002 et juillet 2003 auprès de 40 commerçantes à Naples et à Sousse. Parmi ces femmes, 13 ont fait l'objet d'entretiens semi-directifs répétés et approfondis. L'enquête a été complétée par des suivis de voyage, ainsi que par des séjours en Tunisie. Ces derniers ont donné l'occasion de réaliser des entretiens avec d'autres membres de la famille participant de l'« entreprise familiale ». Cet éclairage sur le point de vue des autres, maris, enfants, mais aussi clients, a été extrêmement utile pour comprendre la place de cette activité dans les parcours sociaux, familiaux et individuels de ces femmes.

3. Les 40 femmes interrogées ont de 22 à 55 ans, pour une moyenne de 39 ans, ce qui est, pour les sociétés maghrébines, un âge relativement avancé. La plupart d'entre elles sont mariées. Quatre sont des filles qui suivent leurs mères. Trois sont grand-mères.

4. Cela ne signifie pas que l'utilité sociale du travail féminin soit toujours reconnue à la mesure de leur participation à la sphère professionnelle (Jomni, 2000).

5. Sabrina se réfère aux taxes appliquées aux produits importés qui augmentent notablement les prix de vente en Tunisie.

6. Sur la valeur de la villa pour les classes moyennes sahéliennes, voir le travail de Ridha Lamine (2000).

7. Le tourisme est, avec l'agriculture, la première source de revenu du pays. Le poids du tourisme au sens large atteignait, en 2000, 18 \% du PIB tunisien (Chaponnière, 2002). Il aurait connu une chute importante en 2002.

8. Parmi les électrons libres, on trouve des jeunes filles de bonne famille qui préparent leurs trousseaux de mariage, des femmes de milieux aisés qui constituent leur garde-robe, ou encore des femmes qui ont un premier emploi dans la fonction publique et exercent le commerce comme activité complémentaire. Karima, aide-soignante dans un hôpital, déclare ainsi : «jusqu’à maintenant, il y a disons $90 \%$ des gens qui travaillent à l'hôpital qui font du commerce comme moi, parce que c'est pas rentable le salaire qu'ils prennent sur un mois, c'est pas rentable, on peut pas continuer le travail avec 300 dinars (environ 190 euros), ça fait rien du tout pour nous. Si l'homme et la femme quand ils sont mariés ils travaillent pas, ils peuvent pas mener une vie normale parce que c'est trop difficile de vivre en Tunisie, tout est cher et les gens veulent vivre à un certain niveau ils ont du mal à y arriver ".

9. Nombre de ces commerçantes dispose d'une patente ou d'une société d'import-export, ce qui facilite l'obtention de visas d'affaires.

10. Terme créole utilisé au Cap-Vert pour désigner ces commerçantes transnationales.

11. Plus généralement, cette interprétation du port du voile se rattache à un ensemble de travaux qui cherche à mettre en évidence combien la signification de cet acte est changeante 
selon les contextes historiques et sociaux (Charrad, 1998 ; Kapchan, 1996 ; Mac Leod, 1991 ; Secor, 2002).

12. On retrouve cette notion de ruse dans la littérature féministe arabo-musulmane, dans laquelle elle est dépouillée de la connotation péjorative qu'elle trouve parfois dans la langue française. On pense par exemple à la ruse de Schéhérazade pour détourner le calife, symbole du pouvoir masculin, de ses pulsions de cruauté (Mernissi, 2001).

13. Il existait déjà des relations d'échange historiques entre la capitale du Mezzogiorno d'Italie et l'Est du Maghreb. Cependant, la vocation de Naples en tant que place d'achat se confirme avec la fermeture des pays traditionnels d'immigration et l'installation d'une communauté maghrébine en Campanie, qui coïncident avec de profondes transformations socio-économiques dans la région (Schmoll, 2001).

14. Il ne faudrait pas interpréter cette situation comme un transfuge d'un mode de vie arabomusulman : Linda Mac Dowell a ainsi mené une analyse intéressante de la dichotomie espace public/espace privé et de leur association avec le binôme femme décente/femme indécente dans les villes occidentales (1999).

15. Sans entrer dans le détail des discussions dont fait l'objet ce concept, on peut dire que le dar al islam correspond aux régions dans lesquelles la religion musulmane exerce une influence forte sur la sphère publique. Du reste, c'est probablement parce que Naples n'appartient pas au dar al islam qu'on ne trouve guère de circulantes algériennes et marocaines sur la place marchande. Les femmes algériennes préfèreraient par exemple se rendre en Turquie ou au Maroc. Cela reflète l'émancipation plus avancée des femmes tunisiennes, mais aussi leurs avantages légaux (la Majalla, le code du statut personnel (1956), est favorable au travail des femmes), par rapport aux Algériennes ou aux Marocaines pour lesquelles un code de la famille particulièrement strict est encore en vigueur (Moudawana, décrets sur le statut personnel, 1957, réformé en 2004, et code de la famille algérien adopté en 1984).

16. Fedime Deli (2002) signale la forte présence de commerçants turcs arabophones originaires de la région orientale de Mardîn dans les boutiques stambouliotes du district de Laleli.

\section{RÉSUMÉS}

Ce texte propose une lecture de genre des circulations commerciales, à partir de l'étude d'un groupe de femmes tunisiennes pratiquant de fréquents voyages d'achat à Naples. Aborder les circulations commerciales selon une telle perspective implique non seulement de considérer les migrantes sous l'angle de leurs multiples positions sociales, mais aussi à la lumière des différents espaces qu'elles parcourent et mettent en relation. Les pratiques de mobilité des femmes tunisiennes, tout comme leurs « savoir-circuler » reflètent leur appartenance de genre, en même temps qu'elles mènent à une reformulation des rôles des hommes et des femmes dans les sphères productive et reproductive. Pour les femmes, en effet, la circulation est mise au profit de stratégies de mobilité socio-économique et d'autonomisation. Toutefois, cela s'effectue au prix de multiples négociations et arrangements au sein du foyer comme sur les routes et sur les places d'achat, qui sont des espaces essentiellement masculins.

Transnational Spatial Practices and Mobility Strategies of Tunisian Female Traders.

The purpose of this article is to provide a gendered interpretation of transnational commerce migration, drawing on an empirical study of Tunisian women who practice frequent buying 
journeys in Naples, Italy. Analysing transnational trade from a gender perspective enables to consider women's lives not only in light of their multiple social positions, but also in light of the multiple spaces they connect each other through their migratory movements. Tunisian traders' mobility practices and their specific 'know-how to circulate' display their gender belonging and positioning. At the same time, these practices are source of empowerment for women, as they lead to a reformulation of women's and men's roles within both the productive and the reproductive spheres. In fact, transnational trade allows women to pursue strategies of social upward mobility and autonomisation. Nevertheless, it has to be noted that these strategies can be performed only through numerous and continuous negotiations, both at the household level and within typically male spaces such as the travel and trading routes and places.

Prácticas espaciales transnacionales y estrategias de movilidad de comerciantes tunecinas. Este texto propone una lectura de género de las circulaciones comerciales a partir del estudio de un grupo de mujeres tunecinas que practican numerosos viajes a Nápoles para comprar. Abordar las circulaciones comerciales según esta perpectiva implica no sólo considerar a las inmigrantes bajo el ángulo de sus múltiples posiciones sociales sino también a partir de los diferentes espacios que dichas mujeres recorren y ligan entre sí. Las prácticas de movilidad de las mujeres tunecinas, así como su « saber-circular » reflejan su pertenencia de género y conducen a la reformulación de los roles de hombres y mujeres en las esferas productiva y reproductiva. Para las mujeres, la circulación forma parte de una estrategia de movilidad socioeconómica y de autonomía. Con todo, el precio a pagar son las negociaciones y los acuerdos múltiples tanto en el hogar como en las rutas y en los lugares de compra, espacios esencialmente masculinos.

Pratiche spaziali trasnazionali e strategie di mobilità delle commercianti tunisine.Questo testo si propone di dimostrare l'interesse di una lettura di genere al fenomeno delle circolazioni commerciali, a partire dallo studio di un gruppo di donne tunisine, che svolgono frequenti viaggi di rifornimento a Napoli. Questo tipo di lettura porta a considerare le donne migranti non solo alla luce delle loro molteplici posizioni sociali, ma anche sotto il profilo dei diversi spazi che esse percorrono e mettono in relazione. Le pratiche di mobilità delle donne tunisine, cosi come il loro "sapere circolatorio", sono il riflesso della loro appartenenza di genere. Allo stesso tempo, queste pratiche portano a una riformulazione dei ruoli degli uomini e delle donne nelle sfere produttive e riproduttive. Da parte delle donne, infatti, la circolazione migratoria è praticata allo scopo di perseguire strategie di mobilità socio-economica e di autonomizzazione. Tuttavia, ciò si realizza al prezzo di numerose e continue negoziazioni, sia nell'ambito del nucleo familiare sia in quello delle vie e dei luoghi d'acquisto, in quanto spazi essenzialmente maschili.

\section{INDEX}

Mots-clés : circulation migratoire, commerçants, femmes, mobilité sociale, relations familiales, territorialité, Tunisiens

Index géographique : Italie

\section{AUTEUR}

\section{CAMILLE SCHMOLL}

Laboratoire Mosaïques, UMR Louest. Actuellement en post-doctorat au département de géographie de l'Università degli Studi di Napoli Federico II. Adresse électronique : camilleschmoll@yahoo.fr. 